\title{
Large Shear Rate Behaviour For the Hébraud-Lequeux Model
}

\author{
Julien OLIVIER*
}

January $2012^{\dagger}$

\begin{abstract}
The Hébraud-Lequeux model is a model describing the flow of soft glassy material in a simple shear flow configuration. It is given by a kinetic/Fokker-Planck type of equation whose coefficients depend on the shear rate of the experiment. In this paper we want to study what happens to the stationary solutions of this model when the shear rate is asymptotically large. In order to that, we expand the solution of the equation using singular perturbation tools. In the end, we rigorously prove the estimate of HÉBRAUD and LEQUEUX that the material asymptotically behaves as a Newtonian fluid.
\end{abstract}

Keywords: Singular limit, Non Newtonian Rheology, Kinetic equations

AMS Subject Classification: 35C20,76A05

\section{Introduction}

We are interested in the behaviour at large shear rates of the Hébraud-Lequeux model (referred to as HL in the sequel for brevity), derived by HÉBraUd and LEQUeux in [?]. This model is a Fokker-Planck-like model which aims at describing the behaviour of a generic soft glassy material. This model has been studied by various authors, both in its nonstationary version and its stationary one. One can cite [?] for a PDE approach of the well-posedness and [?] for a stochastic analysis of the Cauchy problem. The well-posedness of the stationary problem has been adressed in [?] and [?]. The study of the glass transition in this model has been conducted in [?] with a direct approach which is very specific to the $1 d$ setting of the HL model and was revisited in [?] with a more robust method on which this paper is modelled. The need for a robust approach to this question arise when we try to justify a multidimensional model generalizing the one of HÉBRAUD and LEQUeUX in [?].

Let us now review the description of the HL model. In this model the state of a sample of the material undergoing a shear rate $\dot{\gamma}$ is described by means of a

\footnotetext{
*Partially supported by the FP7-REGPOT-2009-1 project "Archimedes Center for Modeling, Analysis and Computation".

${ }^{\dagger}$ The original publication will be available at www.sciencechina.com and www.springerlink.com
} 
probability density $p$ over the stress space. The stress of the sample is noted $\tau$. Because of the elastoviscoplastic nature of soft glassy material, when submitted to a shear rate, we expect it to have a transitory phase followed by a sationary phase. The HL model can handle both regimes but we will be interested only on the final stationary phase. In this phase $p$ is a probability density which follows the following dimensionless equation:

$$
\left\{\begin{array}{l}
-\mu \Gamma \partial_{\sigma}^{2} p+h p+y \partial_{\sigma} p=\Gamma \delta_{0} \\
\int_{\mathbf{R}} p(\sigma) \mathrm{d} \sigma=1 \\
p \geq 0
\end{array}\right.
$$

where $h$ stands for the characteristic function of the set $\mathbf{R} \backslash[-1,1]$. The term $\delta_{0}$ is the Dirac mass. The parameter $y$ is a dimensionless shear rate $\dot{\gamma} / \dot{\gamma}_{c}$ where $\dot{\gamma}_{c}$ is a critical shear rate depending on the material. The positive real number $\mu$ is a dimensionless constant describing the state of the material with respect to the glass transition.

In [?] we proved that when the shear rate is small, that means when $y \ll 1$ we can get various behaviour for the model depending on the value of $\mu$. More precisely, we proved that when $\mu>1 / 2$ the behaviour of the model is at main order the behaviour of a Newtonian fluid, when $\mu<1 / 2$ the behaviour is the one of a threshold fluid of Hershell-Bulkley type and when $\mu=1 / 2$ we have a power-law fluid with exponent $1 / 5$. In the present paper we are interested in the large shear rate behaviour of the fluid, that is to say $y \gg 1$ and we will prove that the behaviour is the one of a Newtonian fluid independently of the value of $\mu$, which is the experimental behaviour expected for soft glassy materials [?].

Finally $\Gamma$ is called the fluidity and is related to the integral constraint $\int p=1$. We can see by integrating the differential equation that

$$
\Gamma=\int_{|\sigma|>1} p(\sigma) \mathrm{d} \sigma .
$$

Because $p$ is a probability density, $\Gamma$ is none other than the probability to find $|\sigma|>1$ and thus

$$
0 \leq \Gamma \leq 1
$$

The stress of a sample of material in this model is recovered by

$$
\tau=\int_{\mathbf{R}} \sigma p(\sigma) \mathrm{d} \sigma
$$

Our aim is to study the link between $y$ which is given and $\tau$ which is computed via (??) where $p$ is the solution of (??). We have already done so in the limiting case $y \rightarrow 0$ in another paper [?] (with M. RENARDY) and wish to understand the other limiting case $y \rightarrow+\infty$. For the sequel of the paper we note $y=1 / \varepsilon$.

We are thus interested in the behaviour of an elliptic ODE with a perturbation of the form $\partial_{\sigma} p / \varepsilon$. This problem often arises in the study of the asymptotic behaviour of various models. One can refer for instance to the vanishing Rossby number approximation in oceanography [?]. What is specific in our model is first that it is set in an unbounded domain while the singular limits we mentionned are set in bounded domains. The consequence is that, if we try to take the naï ve limit $\varepsilon \rightarrow 0$, we end up with the limit equation $\partial_{\sigma} p=0$ which has 
only 0 as a solution on an unbounded domain. This limit is not rich enough to describe the limiting behaviour of the model. Moreover, the second specific feature of our model is that we have $\int p=1$ which is true for all $\varepsilon>0$ and must remain true at the limit. This is clearly incompatible with the naïve limit $p=0$ that we just mentionned. This difficulty is related to the fact that, despite appearances, our problem is truly nonlinear and the integral constraint makes System (??) quite rigid.

To overcome these difficulties we must describe the solution of (??) more accurately than with just the limit. This is why we decompose the solution into three parts corresponding to the intervals $]-\infty, 0[] 0,,1[$ and $] 1,+\infty[$ completed with transmission conditions at 0 and 1 which are given by the expected global regularity of the solution of (??). We will see that these three parts behave very differently when $\varepsilon \rightarrow 0$. The situation is a bit different from the limit $y \rightarrow 0$ we studied in [?] for which we divided in $]-\infty,-1[]-1,,1[$ and $] 1,+\infty[$ in order to study the passage from a density with support in $\mathbf{R}$ to a density with support in $]-1,1[$.

To conlude this introduction we would also like to point out that we used here a standard method of a priori estimates on the remainder of the expansion of $p$ to prove the "convergence" while in [?] we took advantage of the $1 d$ setting to use the singular perturbation theory of [?] and get directly the convergence of the asymptotic expansion. The method used in this paper can, however, be extended fairly straightforwardly, for instance to multi dimensional version of the HL model.

\section{Main Results}

Let us first recall the well-posedness theo on which the sequel of the study relies on:

Theorem 1. The stationary Hébraud-Lequeux model,

$$
\left\{\begin{array}{l}
-\mu \Gamma \partial_{\sigma}^{2} p+h p+\frac{1}{\varepsilon} \partial_{\sigma} p=\Gamma \delta_{0} \\
\int_{\mathbf{R}} p(\sigma) \mathrm{d} \sigma=1
\end{array}\right.
$$

has a unique solution $p \in \mathrm{H}^{1}(\mathbf{R})$ which decays exponentially when $|\sigma| \rightarrow+\infty$, for every $\varepsilon>0$.

For the proof we refer to [?, ?]. We can now state the main result of this paper:

Theorem 2. Let us note $p$ the solution to (??) and $\tau$ given by (??) then for $\varepsilon \rightarrow 0$ we have

$$
\tau \sim \frac{1}{\varepsilon},
$$

or more precisely

$$
\tau=\frac{1}{\varepsilon}+\mathcal{O}(1) .
$$


For the material, this means that when the shear rate is large the rheological law is at main order the law of Newtonian fluid with a viscosity independent of $\mu$. This theo is the main goal of this paper but it will need some intermediate results. The main idea which we have already used in [?] is to work directly on $p$ instead of working on $\tau$. For $p$ we have the following asymptotic result:

Proposition 1. The solution $p$ to the HL problem given by theo ?? can be expanded when $\varepsilon \rightarrow 0$ as $p=p^{\mathrm{app}}+p^{\mathrm{rem}}$, where $p^{\mathrm{app}}$ has the following expression:

$$
p^{\mathrm{app}}= \begin{cases}\varepsilon \exp \left(\frac{\sigma}{\mu \varepsilon}\right) & \text { if } \sigma \leq 0, \\ \varepsilon & \text { if } 0 \leq \sigma \leq 1, \\ \varepsilon \exp (-\varepsilon(\sigma-1)) & \text { if } 1 \leq \sigma,\end{cases}
$$

and $p^{\text {rem }}$ verifies the following estimates:

$$
\begin{aligned}
\left\|\exp \left(-\frac{\cdot}{2 \mu \varepsilon}\right) p^{\mathrm{rem}}\right\|_{\mathrm{L}^{2}(]-\infty, 0[)} & =\mathcal{O}\left(\varepsilon^{5 / 2}\right), \\
\left\|p^{\mathrm{rem}}\right\|_{L^{2}(] 0,1[)} & =\mathcal{O}\left(\varepsilon^{3 / 2}\right), \\
\| \exp \left(\frac{\varepsilon}{2}(\cdot-1) p^{\mathrm{rem}} \|_{L^{2}(] 1,+\infty[)}\right. & =\mathcal{O}\left(\varepsilon^{3 / 2}\right) .
\end{aligned}
$$

This asymptotic expansion relies on the following estimate on $\Gamma$ :

Lemma 1. The following expansion of the fluidity $\Gamma$ is true:

$$
\Gamma=1+\mathcal{O}(\varepsilon)
$$

This lemma only requires $p^{\text {app }}$. The scheme of proof is then the following:

1. Compute formally the main order of $p, p^{\text {app }}$ and the remainder problem (??).

2. Prove Proposition ?? assuming Lemma ??. This justifies the formal asymptotic expansion and also gives estimate on the remainder $p^{\text {rem }}$.

3. Prove theo ??

4. Prove Lemma ??.

The organization of the paper follows the scheme of proof: we carry out the formal expansions in Section ?? and give the remainder problem in Section ??. Then we prove inequalities (??)-(??) assuming Lemma ??. Then we use the estimates obtained to prove the theo in Section ??. Finally, to complete the proof, we show Lemma ?? in Section ??. We prefered to delay the proof of Lemma ?? because it is a bit long and we did not wish to interrupt the proof of theo ??.

\section{Formal Expansions}

Since we are interested in what happens to the system when $y$ is large, we set $y=1 / \varepsilon$ and study the behaviour as $\varepsilon \rightarrow 0^{+}$. As we did before for the low shear rate asymptotics, we use asymptotic expansions on $p$ instead of working directly on $\tau$. We get these asymptotics in a suitable space so that we can go from $p$ to $\tau$ just by integrating the expansion of $p$ against $\sigma$. 


\subsection{Rewriting of the System}

We are interested in the limit behaviour as $\varepsilon \rightarrow 0$ of the model given by (??):

$$
\left\{\begin{array}{l}
-\mu \Gamma \partial_{\sigma}^{2} p+h p+\frac{1}{\varepsilon} \partial_{\sigma} p=\Gamma \delta_{0}, \\
\int_{\mathbf{R}} p(\sigma) \mathrm{d} \sigma=1 .
\end{array}\right.
$$

For this study we have to rewrite the equations in terms of what happens on $\mathbf{R}_{-,}^{*}[0,1]$ and $] 1,+\infty[$. We note

$$
p_{-}=p_{\mid \mathbf{R}_{-}^{*}}, \quad p_{+, i}=p_{\mid[0,1]} \quad p_{+, e}=p_{\mid[1,+\infty[} \cdot
$$

We rewrite this system in terms of these new variables:

$$
\left\{\begin{array}{l}
-\mu \Gamma \partial_{\sigma}^{2} p_{-}+h p_{-}+\frac{1}{\varepsilon} \partial_{\sigma} p_{-}=0 \\
-\mu \Gamma \partial_{\sigma}^{2} p_{+, i}+\frac{1}{\varepsilon} \partial_{\sigma} p_{+, i}=0 \\
-\mu \Gamma \partial_{\sigma}^{2} p_{+, e}+p_{+, e}+\frac{1}{\varepsilon} \partial_{\sigma} p_{+, e}=0 \\
p_{+, i}(0)=p_{-}(0) \\
p_{+, e}(1)=p_{-, i}(1), \\
\partial_{\sigma} p_{+, i}(0)=\partial_{\sigma} p_{-}(0)-\frac{1}{\mu} \\
\partial_{\sigma} p_{+, e}(1)=\partial_{\sigma} p_{+, i}(1) \\
\int_{-\infty}^{0} p_{-}(\sigma) \mathrm{d} \sigma+\int_{0}^{1} p_{+, i}(\sigma) \mathrm{d} \sigma+\int_{1}^{+\infty} p_{+, e}(\sigma) \mathrm{d} \sigma=1
\end{array}\right.
$$

Transmission conditions

$$
\begin{aligned}
& p_{+, i}(0)=p_{-}(0), \\
& p_{+, e}(1)=p_{-, i}(1),
\end{aligned}
$$

come from the fact that $p$ is continuous. The condition

$$
\partial_{\sigma} p_{+, i}(0)=\partial_{\sigma} p_{-}(0)-\frac{1}{\mu},
$$

takes into account the Dirac mass at 0 while the last transmission condition

$$
\partial_{\sigma} p_{+, e}(1)=\partial_{\sigma} p_{+, i}(1)
$$

comes from the fact that we assume $\partial_{\sigma} p$ to be continuous at 1 (nothing in (??) could balance a jump in the derivative at 1). Now we change variables to find the right profiles equation. We set

$$
\begin{array}{rlrl}
p_{-}(\sigma) & =q_{-}\left(\frac{\sigma}{\varepsilon}\right), & p_{+, i}(\sigma)=q_{+, i}(\sigma)+q_{b l}\left(\frac{1-\sigma}{\varepsilon}\right), \\
p_{+, e}(\sigma) & =q_{+, e}(\varepsilon(\sigma-1)),
\end{array}
$$


and we note $z$ the variable of $q_{-}, q_{b l}$ and $q_{+, e}$ (and also the one of $q_{+, i}$ for simplicity). As $q_{b l}$ describes a boundary layer in the neighbourhood of $\sigma=1$ we assume exponential decay of $q_{b l}$ and all its derivatives for all $z \neq 0$. We finally write the system which will allow us to compute the equations of the profile:

$$
\left\{\begin{array}{l}
-\frac{1}{\varepsilon^{2}} \mu \Gamma \partial_{z}^{2} q_{-}+h_{\varepsilon} q_{-}+\frac{1}{\varepsilon^{2}} \partial_{z} q_{-}=0, \\
-\mu \Gamma \partial_{z}^{2} q_{+, i}+\frac{1}{\varepsilon} \partial_{z} q_{+, i}=0, \\
-\mu \Gamma \partial_{z}^{2} q_{b l}-\partial_{z} q_{b l}=0, \\
-\mu \Gamma \varepsilon^{2} \partial_{z}^{2} q_{+, e}+q_{+, e}+\partial_{z} q_{+, e}=0, \\
q_{+, i}(0)+q_{b l}\left(\frac{1}{\varepsilon}\right)=q_{-}(0), \\
q_{+, e}(0)=q_{+, i}(1)+q_{b l}(0), \\
\partial_{z} q_{+, i}(0)-\frac{1}{\varepsilon} \partial_{z} q_{b l}\left(\frac{1}{\varepsilon}\right)=\frac{1}{\varepsilon} \partial_{z} q_{-}(0)-\frac{1}{\mu}, \\
\partial_{z} q_{+, e}(0)=\frac{1}{\varepsilon} \partial_{z} q_{+, i}(1)-\frac{1}{\varepsilon^{2}} \partial_{z} q_{b l}(0), \\
\varepsilon \int_{-\infty}^{0} q_{-}(z) \mathrm{d} z+\int_{0}^{1} q_{+, i}(z) \mathrm{d} z+\varepsilon \int_{0}^{1 / \varepsilon} q_{b l}(z) \mathrm{d} z+\frac{1}{\varepsilon} \int_{0}^{+\infty} q_{+, e}(z) \mathrm{d} z=1,
\end{array}\right.
$$

where we have defined

$$
h_{\varepsilon}(z)=h(\varepsilon z) .
$$

Note that since $h=\mathbf{1}_{\mathbf{R} \backslash[-1,1]}$, we have

$$
h_{\varepsilon}(z)= \begin{cases}1 & \text { if }|z| \geq \frac{1}{\varepsilon} \\ 0 & \text { otherwise }\end{cases}
$$

Now if the expansion we make is ever to be valid we must have $q_{-}$exponentially decreasing when $z$ decreases to $-\infty$. Then if we consider the function $z \mapsto h_{\varepsilon}(z) \exp (z)$ then it is easy to see that it is exponentially small (ie $\sim \exp (-\lambda / \varepsilon)$ for some $\lambda>0)$ in any norm we could consider. Thus in the following we will treat all terms multiplied by $h_{\varepsilon}$ to be smaller than any $\varepsilon^{k}$ for any $k \geq 0$. As a remark we cannot do this on the other side and have to break it into the interior part $q_{+, i}$ and the exterior part $q_{+, e}$.

\subsection{Ansaetze}

We make the following ansaetze:

$$
\begin{aligned}
q_{-} & =\varepsilon q_{-}^{1}+\varepsilon^{2} q_{-}^{2}+\varepsilon^{3} q_{-}^{3}+\ldots, \\
q_{+, i} & =\varepsilon q_{+, i}^{1}+\varepsilon^{2} q_{+, i}^{2}+\varepsilon^{3} q_{+, i}^{3}+\ldots, \\
q_{b l} & =\varepsilon^{3} q_{b l}^{3}+\varepsilon^{4} q_{b l}^{4}+\ldots, \\
q_{+, e} & =\varepsilon q_{+, e}^{1}+\varepsilon^{2} q_{+, e}^{2}+\varepsilon^{3} q_{+, e}^{3} \ldots
\end{aligned}
$$

Of course we could have put terms of order 0 for $q_{-}, q_{+, i}$ and $q_{+, e}$ and terms of order 0,1 and 2 for $q_{b l}$. When one does that, by putting the ansaetze in the 
equations of (??), we would see that these terms must be 0 ; because they solve linear Dirichlet problems with no exterior forces. We recall that we have (??) which is the following consistency equation between $\Gamma$ and $p$ :

$$
\Gamma=\int_{|\sigma|>1} p(\sigma) \mathrm{d} \sigma
$$

which can be rewritten in terms of $q_{-}$and $q_{+, e}$

$$
\Gamma=\frac{1}{\varepsilon} \int_{0}^{+\infty} q_{+, e}(z) \mathrm{d} z+\varepsilon \int_{-\infty}^{-1 / \varepsilon} q_{-}(z) \mathrm{d} z .
$$

With such an expression, if we assume enough regularity of the profiles (which will be checked after we have computed them), we can derive the following ansatz on $\Gamma$ :

$$
\Gamma=c_{0}+c_{1} \varepsilon+c_{2} \varepsilon^{2}+c_{3} \varepsilon^{3} \ldots
$$

with $\forall k, c_{k}=\int_{0}^{+\infty} q_{+, e}^{k+1}(z) \mathrm{d} z$ because, as we remarked, the second term is exponentially small.

\subsection{Profiles}

In this section we compute the profiles $q_{-}^{k}, q_{+, i}^{k}$ and $q_{+, e}^{k}$ for $k=1,2$ even if, in the end, we will only use the approximation given by the terms of order 1 . Indeed, it can be interesting to compare the terms of order 2, that we give here, to the approximation of (??) given in (??).

All we need to do is to plug (??), (??) and (??) in (??) and identify the formal powers of $\varepsilon$.

Order $\varepsilon$.

We first look for $q_{+, e}^{1}$ :

$$
\left\{\begin{array}{l}
\partial_{z} q_{+, e}^{1}+q_{+, e}^{1}=0 \\
\int_{0}^{+\infty} q_{+, e}^{1}(z) \mathrm{d} z=1
\end{array}\right.
$$

which leads to

$$
q_{+, e}^{1}(z)=\exp (-z) .
$$

We also have that $c_{0}=1$. Now we can look for $q_{+, i}^{1}$ which solves the following problem

$$
\left\{\begin{array}{l}
\partial_{z} q_{+, i}^{1}=0 \\
q_{+, i}^{1}(1)=1
\end{array}\right.
$$

whose solution is obviously given by

$$
q_{+, i}^{1}=1
$$

identically. Finally we compute for $q_{-}^{1}$ which solves the problem:

$$
\left\{\begin{array}{l}
-\mu \partial_{z}^{2} q_{-}^{1}+\partial_{z} q_{-}^{1}=0, \\
q_{-}^{1}(0)=1, \\
\partial_{z} q_{-}^{1}(0)=\frac{1}{\mu}
\end{array}\right.
$$


whose solution is

$$
q_{-}^{1}(z)=\exp (z / \mu)
$$

$\operatorname{Order} \varepsilon^{2}$. Once again we start from the right:

$$
\left\{\begin{array}{l}
\partial_{z} q_{+, e}^{2}+q_{+, e}^{2}=0 \\
\int_{0}^{+\infty} q_{+}^{2}(z) \mathrm{d} z=-\int_{0}^{1} q_{+, i}^{1}(z) \mathrm{d} z=-1,
\end{array}\right.
$$

whose solution is $q_{+, e}^{2}(z)=-\exp (-z)$. We also have $c_{1}=-1$. We continue by correcting the derivative of the previous approximation with a boundary layer:

$$
\left\{\begin{array}{l}
-\mu \partial_{z}^{2} q_{b l}^{3}-\partial_{z} q_{b l}^{3}=0, \\
\partial_{z} q_{b l}^{3}(0)=-\partial_{z} q_{+, e}^{1}(0)=-(-1),
\end{array}\right.
$$

for which we have the solution $q_{b l}^{3}(z)=-\mu \exp (-z / \mu)+1$ up to an additive constant. We choose to take this solution so that $q_{b l}^{3}(0)=0$. Only now do we look for $q_{+, i}^{2}$ :

$$
\left\{\begin{array}{l}
\partial_{z} q_{+, i}^{2}=0, \\
q_{+, i}^{2}(1)=-1,
\end{array}\right.
$$

whose solution is $q_{+, i}^{2}=-1$. Now we can find the profile $q_{-}^{2}$ by solving the problem

$$
\left\{\begin{array}{l}
-\mu \partial_{z}^{2} q_{-}^{2}+\partial_{z} q_{-}^{2}=-\mu \partial_{z}^{2} q_{-}^{1}, \\
q_{-}^{2}(0)=-1 \\
\partial_{z} q_{-}^{2}(0)=0
\end{array}\right.
$$

whose solution is $q_{-}^{2}(z)=(z / \mu-1) \exp (z / \mu)$.

\section{Proof of Proposition ??}

To construct the main order of $p$ in its behaviour as $\varepsilon \rightarrow 0$ we simply truncate the expansions of (??)-(??) at first order, replace $q_{-}^{1}, q_{+, i}^{1}$ and $q_{+, e}^{1}$ by the expressions given at (??),(??) and (??). We introduce the notation:

$$
\begin{aligned}
& p_{-}^{\mathrm{app}}(\sigma)=\varepsilon q_{-}^{1}\left(\frac{\sigma}{\varepsilon}\right), \\
& p_{+, i}^{\mathrm{app}}(\sigma)=\varepsilon q_{+, i}^{1}(\sigma)+\varepsilon^{2} q_{+, i}^{2}(\sigma), \\
& p_{+, e}^{\mathrm{app}}(\sigma)=\varepsilon q_{+, e}^{1}(\varepsilon(\sigma-1)),
\end{aligned}
$$

and this gives the expression of $p^{\text {app }}$ given in (??). Finally, we note $p_{-}^{\text {rem }}=$ $p_{-}-p_{-}^{\mathrm{app}}, p_{+, i}^{\mathrm{rem}}=p_{+, i}-p_{+, i}^{\mathrm{app}}$ and $p_{+, e}^{\mathrm{rem}}=p_{+, e}-p_{+, e}^{\mathrm{app}}$. We write the problem 
solved by the remainder terms:

$$
\left\{\begin{array}{l}
-\mu \Gamma \partial_{\sigma}^{2} p_{-}^{\mathrm{rem}}+h p_{-}^{\mathrm{rem}}+\frac{1}{\varepsilon} \partial_{\sigma} p_{-}^{\mathrm{rem}}=R_{-}, \\
-\mu \Gamma \partial_{\sigma}^{2} p_{+, i}^{\mathrm{rem}}+\frac{1}{\varepsilon} \partial_{\sigma} p_{+, i}^{\mathrm{rem}}=0, \\
-\mu \Gamma \partial_{\sigma}^{2} p_{+, e}^{\mathrm{rem}}+p_{+, e}^{\mathrm{rem}}+\frac{1}{\varepsilon} \partial_{\sigma} p_{+, e}^{\mathrm{rem}}=R_{+, e} \\
p_{+, i}^{\mathrm{rem}}(0)=p_{-}^{\mathrm{rem}}(0) \\
p_{+, e}^{\mathrm{rem}}(1)=p_{-, i}^{\mathrm{rem}}(1) \\
\partial_{\sigma} p_{+, i}^{\mathrm{rem}}(0)=\partial_{\sigma} p_{-}^{\mathrm{rem}}(0), \\
\partial_{\sigma} p_{+, e}^{\mathrm{rem}}(1)-\varepsilon^{2}=\partial_{\sigma} p_{+, i}^{\mathrm{rem}}(1), \\
\int_{-\infty}^{0} p_{-}^{\mathrm{rem}}(\sigma) \mathrm{d} \sigma+\int_{0}^{1} p_{+, i}^{\mathrm{rem}}(\sigma) \mathrm{d} \sigma+\int_{1}^{+\infty} p_{+, e}^{\mathrm{rem}}(\sigma) \mathrm{d} \sigma=-\varepsilon+\mu \varepsilon^{2}
\end{array}\right.
$$

where we have set

$$
\begin{aligned}
R_{-} & =\mu \Gamma \partial_{\sigma}^{2} p_{-}^{\mathrm{app}}-h p_{-}^{\mathrm{app}}-\frac{1}{\varepsilon} \partial_{\sigma} p_{-}^{\mathrm{app}} \\
& =\left(\frac{\Gamma-1}{\mu \varepsilon}-\varepsilon h\right) \exp \left(\frac{\sigma}{\mu \varepsilon}\right), \\
R_{+, e} & =\mu \Gamma \partial_{\sigma}^{2} p_{+, e}^{\mathrm{app}}-p_{+, e}^{\mathrm{app}}-\frac{1}{\varepsilon} \partial_{\sigma} p_{+, e}^{\mathrm{app}} \\
& =\mu \Gamma \varepsilon^{3} \exp (-\varepsilon(\sigma-1)) .
\end{aligned}
$$

Note, for example, that we obtain the last transmission condition by writing:

$$
\partial_{\sigma} p_{+, e}(1)=\partial_{\sigma} p_{+, i}(1)
$$

which becomes, using $p=p^{\text {app }}+p^{\text {rem }}$,

$$
\partial_{\sigma} p_{+, e}^{\mathrm{rem}}(1)+\partial_{\sigma} p_{+, e}^{\mathrm{app}}(1)=\partial_{\sigma} p_{+, i}^{\mathrm{rem}}(1)+\partial_{\sigma} p_{+, i}^{\mathrm{app}}(1),
$$

and thus, using the expressions of $p_{+, e}^{\text {app }}$ and $p_{+, i}^{\text {app }}$,

$$
\partial_{\sigma} p_{+, e}^{\mathrm{rem}}(1)-\varepsilon^{2}=\partial_{\sigma} p_{+, i}^{\mathrm{rem}}(1) .
$$

We will now prove the estimates (??)-(??) of Proposition ??. It is useful to introduce $\widetilde{p}_{-}^{\text {rem }}$ and $\widetilde{p}_{+, e}^{\text {rem }}$ defined through,

$$
\begin{aligned}
& p_{-}^{\mathrm{rem}}=\exp \left(\frac{\sigma}{2 \mu \varepsilon}\right) \widehat{p}_{-}^{\mathrm{rem}}, \\
& p_{+, e}^{\mathrm{rem}}=\exp \left(-\frac{\varepsilon}{2}(\sigma-1)\right) \widetilde{p}_{+, e}^{\mathrm{rem}} .
\end{aligned}
$$

This allows us to take advantage of the exponential decay of the right-hand sides of (??) and explains the form of estimates (??) and (??): we are going to show that $\widetilde{p}_{-}^{\text {rem }}$ and $\widetilde{p}_{+, e}^{\text {rem }}$ are bounded in $L^{2}(]-\infty, 0[)$ and $L^{2}(] 1,+\infty[)$ respectively. 
We thus now rewrite the system we will study with the unknowns $\widetilde{p}_{-}^{\mathrm{rem}}, p_{+, i}^{\mathrm{rem}}$ and $\widetilde{p}_{+, e}^{\text {rem }}$

$$
\begin{aligned}
& \partial_{\sigma} p_{-}^{\mathrm{rem}}(\sigma)=\exp \left(\frac{\sigma}{2 \mu \varepsilon}\right)\left(\partial_{\sigma} \widetilde{p}_{-}^{\mathrm{rem}}(\sigma)+\frac{1}{2 \mu \varepsilon} \widetilde{p}_{-}^{\mathrm{rem}}(\sigma)\right), \\
& \partial_{\sigma}^{2} p_{-}^{\mathrm{rem}}(\sigma)=\exp \left(\frac{\sigma}{2 \mu \varepsilon}\right)\left(\partial_{\sigma}^{2} \widetilde{p}_{-}^{\mathrm{rem}}(\sigma)+\frac{1}{\mu \varepsilon} \partial_{\sigma} \widetilde{p}_{-}^{\mathrm{rem}}(\sigma)+\frac{1}{4 \mu^{2} \varepsilon^{2}} \widetilde{p}_{-}^{\mathrm{rem}}(\sigma)\right), \\
& \partial_{\sigma} p_{+, e}^{\mathrm{rem}}(\sigma)=\exp \left(-\frac{\varepsilon}{2}(\sigma-1)\right)\left(\partial_{\sigma} \widetilde{p}_{+, e}^{\mathrm{rem}}(\sigma)-\frac{\varepsilon}{2} \widetilde{p}_{+, e}^{\mathrm{rem}}(\sigma)\right), \\
& \partial_{\sigma}^{2} p_{+, e}^{\mathrm{rem}}(\sigma)=\exp \left(-\frac{\varepsilon}{2}(\sigma-1)\right)\left(\partial_{\sigma}^{2} \widetilde{p}_{+, e}^{\mathrm{rem}}(\sigma)+\varepsilon \partial_{\sigma} \widetilde{p}_{+, e}^{\mathrm{rem}}(\sigma)+\frac{\varepsilon^{2}}{4} \widetilde{p}_{+, e}^{\mathrm{rem}}(\sigma)\right) .
\end{aligned}
$$

We then replace in (??) and simplify both sides by the appropriate exponential:

$$
\left\{\begin{array}{l}
-\mu \Gamma \partial_{\sigma}^{2} \widetilde{p}_{-}^{\mathrm{rem}}+\left(h+\frac{1}{2 \mu \varepsilon^{2}}\left(1-\frac{\Gamma}{2}\right)\right) \widetilde{p}_{-}^{\mathrm{rem}}+\frac{1-\Gamma}{\varepsilon} \partial_{\sigma} \widetilde{p}_{-}^{\mathrm{rem}}=\widehat{R}_{-} \\
-\mu \Gamma \partial_{\sigma}^{2} p_{+, i}^{\mathrm{rem}}+\frac{1}{\varepsilon} \partial_{\sigma} p_{+, i}^{\mathrm{rem}}=0 \\
-\mu \Gamma \partial_{\sigma}^{2} \widetilde{p}_{+, e}^{\mathrm{rem}}+\left(\frac{1}{2}-\frac{\mu \Gamma \varepsilon^{2}}{4}\right) \widetilde{p}_{+, e}^{\mathrm{rem}}+\left(\frac{1}{\varepsilon}-\mu \Gamma \varepsilon\right) \partial_{\sigma} p_{+, e}^{\mathrm{rem}}=\widehat{R}_{+, e} \\
p_{+, i}^{\mathrm{rem}}(0)=\widetilde{p}_{-}^{\mathrm{rem}}(0) \\
\widetilde{p}_{+, e}^{\mathrm{rem}}(1)=p_{-, i}^{\mathrm{rem}}(1) \\
\partial_{\sigma} p_{+, i}^{\mathrm{rem}}(0)=\partial_{\sigma} \widetilde{p}_{-}^{\mathrm{rem}}(0)+\frac{1}{2 \mu \varepsilon} \widetilde{p}_{-}^{\mathrm{rem}}(0) \\
\partial_{\sigma} \widetilde{p}_{+, e}^{\mathrm{rem}}(1)-\frac{\varepsilon}{2} \widetilde{p}_{+, e}^{\mathrm{rem}}-\varepsilon^{2}=\partial_{\sigma} p_{+, i}^{\mathrm{rem}}(1)
\end{array}\right.
$$

where

$$
\begin{aligned}
& \widehat{R}_{-}=R_{-} \exp \left(-\frac{\sigma}{2 \mu \varepsilon}\right)=\left(\frac{\Gamma-1}{\mu \varepsilon}-\varepsilon h\right) \exp \left(\frac{\sigma}{2 \mu \varepsilon}\right), \\
& \widehat{R}_{+, e}=R_{+, e} \exp \left(\frac{\varepsilon}{2}(\sigma-1)\right)=\mu \Gamma \varepsilon^{3} \exp \left(-\frac{\varepsilon}{2}(\sigma-1)\right) .
\end{aligned}
$$

We do an energy estimate on System (??). We multiply each equation by its unknown, integrate on its domain and sum all the obtained equalities: 


$$
\begin{gathered}
\mu \Gamma\left(\int_{-\infty}^{0}\left(\partial_{\sigma} \widetilde{p}_{-}^{\mathrm{rem}}\right)^{2}+\int_{0}^{1}\left(\partial_{\sigma} p_{+, i}^{\mathrm{rem}}\right)^{2}+\int_{1}^{+\infty}\left(\partial_{\sigma} \widetilde{p}_{+, e}^{\mathrm{rem}}\right)^{2}\right) \\
+\int_{-\infty}^{0}\left(h+\frac{1}{2 \mu \varepsilon^{2}}\left(1-\frac{\Gamma}{2}\right)\right)\left(\widetilde{p}_{-}^{\mathrm{rem}}\right)^{2}+\int_{1}^{+\infty}\left(\frac{1}{2}-\frac{\mu \Gamma \varepsilon^{2}}{4}\right)\left(\widetilde{p}_{+, e}^{\mathrm{rem}}\right)^{2} \\
-\mu \Gamma \partial_{\sigma} \widetilde{p}_{-}^{\mathrm{rem}}(0) \widetilde{p}_{-}^{\mathrm{rem}}(0)+\frac{\Gamma-1}{\varepsilon} \frac{p_{-}^{\mathrm{rem}}(0)^{2}}{2} \\
\left.-\mu \Gamma \partial_{\sigma} p_{+, i}^{\mathrm{rem}}(1) p_{+, i}^{\mathrm{rem}}(1)+\mu \Gamma \partial_{\sigma} p_{+, i}^{\mathrm{rem}}(0) p_{+, i}^{\mathrm{rem}}(0)+\frac{1}{\varepsilon} \frac{p_{+, i}^{\mathrm{rem}}(1)^{2}}{2}-\frac{p_{+, i}^{\mathrm{rem}}(0)^{2}}{2}\right) \\
\mu \Gamma \partial_{\sigma} \widetilde{p}_{+, e}^{\mathrm{rem}}(1) \widetilde{p}_{+, e}^{\mathrm{rem}}(1)-\left(\frac{1}{\varepsilon}-\mu \Gamma \varepsilon\right) \frac{\widetilde{p}_{+, e}^{\mathrm{rem}}(1)^{2}}{2} \\
=\int_{-\infty}^{0} \widehat{R}_{-} \widetilde{p}_{-}^{\mathrm{rem}}+\int_{1}^{+\infty} \widehat{R}_{+, e} .
\end{gathered}
$$

Then we use the various transmission conditions to simplify the left-hand side. For example we have:

$$
\begin{aligned}
& -\mu \Gamma \partial_{\sigma} \widetilde{p}_{-}^{\mathrm{rem}}(0) \widetilde{p}_{-}^{\mathrm{rem}}(0)+\frac{1-\Gamma}{\varepsilon} \frac{p_{-}^{\mathrm{rem}}(0)^{2}}{2}+\mu \Gamma \partial_{\sigma} p_{+, i}^{\mathrm{rem}}(0) p_{+, i}^{\mathrm{rem}}(0)-\frac{1}{\varepsilon} \frac{p_{+, i}^{\mathrm{rem}}(0)^{2}}{2} \\
= & \mu \Gamma \widetilde{p}_{-}^{\mathrm{rem}}(0)\left(\partial_{\sigma} p_{+, i}^{\mathrm{rem}}(0)-\partial_{\sigma} \widetilde{p}^{\mathrm{rem}}(0)\right)+\left(\frac{1-\Gamma}{\varepsilon}-\frac{1}{\varepsilon}\right) \frac{p_{-}^{\mathrm{rem}}(0)^{2}}{2} \\
= & \mu \Gamma \widetilde{p}_{-}^{\mathrm{rem}}(0)\left(\frac{1}{\mu \varepsilon} \widetilde{p}_{-}^{\mathrm{rem}}(0)\right)-\frac{\Gamma \frac{p_{-}^{\mathrm{rem}}(0)^{2}}{\varepsilon}}{2}
\end{aligned}
$$

$=0$.

In the same way, the interface terms at $\sigma=1$ simplify except for a term $\mu \Gamma \varepsilon^{2} \widetilde{p}_{+, e}^{\mathrm{rem}}(1)$ which comes from the discrepancy between the derivatives $\partial_{\sigma} p_{+, e}^{\mathrm{rem}}(1)$ and $\partial_{\sigma} p_{+, i}^{\text {rem }}(1)$. This term is actually of a high enough order for our purpose. Then we have the following inequality:

$$
\begin{array}{r}
\mu \Gamma\left(\int_{-\infty}^{0}\left(\partial_{\sigma} \widetilde{p}_{-}^{\mathrm{rem}}\right)^{2}+\int_{0}^{1}\left(\partial_{\sigma} p_{+, i}^{\mathrm{rem}}\right)^{2}+\int_{1}^{+\infty}\left(\partial_{\sigma} \widetilde{p}_{+, e}^{\mathrm{rem}}\right)^{2}\right) \\
+\int_{-\infty}^{0}\left(h+\frac{1}{2 \mu \varepsilon^{2}}\left(1-\frac{\Gamma}{2}\right)\right)\left(\widetilde{p}_{-}^{\mathrm{rem}}\right)^{2}+\int_{1}^{+\infty}\left(\frac{1}{2}-\frac{\mu \Gamma \varepsilon^{2}}{4}\right)\left(\widetilde{p}_{+, e}^{\mathrm{rem}}\right)^{2} \\
\leq \mu \Gamma \varepsilon^{2}\left|\widetilde{p}_{+, e}^{\mathrm{rem}}(1)\right|+\left|\int_{-\infty}^{0} \widehat{R}_{-} \widetilde{p}_{-}^{\mathrm{rem}}\right|+\left|\int_{1}^{+\infty} \widehat{R}_{+, e} \widetilde{p}_{+, e}^{\mathrm{rem}}\right| .
\end{array}
$$

Our goal is to bound by above every term in the right-hand side by terms appearing in the left hand side and by quantities independent of $p^{\text {rem }}$. Let us assume for now Lemma ?? which will be proved in Section ??. Recall also that we have $\Gamma \leq 1$ from (??).

Bound for $\left|\int_{1}^{+\infty} \widehat{R}_{+, e} \widetilde{p}_{+, e}^{\text {rem }}\right|$. 
Firstly we have by (??) and a direct computation of the integral that:

$$
\left\|\widehat{R}_{+, e}\right\|_{\mathrm{L}^{2}(] 1,+\infty[)}=\mathcal{O}\left(\varepsilon^{5 / 2}\right),
$$

so that by Hölder and Young's inequality one may write:

$$
\begin{aligned}
\left|\int_{1}^{+\infty} \widehat{R}_{+, e} \widetilde{p}_{+, e}^{\mathrm{rem}}\right| & \leq\left\|\widehat{R}_{+, e}\right\|_{\mathrm{L}^{2}(] 1,+\infty[)}\left\|\widehat{p}_{+, e}^{\mathrm{rem}}\right\|_{\mathrm{L}^{2}(] 1,+\infty[)} \\
& \leq\left(\frac{1}{\varepsilon^{1 / 2}}\left\|\widehat{R}_{+, e}\right\|_{\mathrm{L}^{2}(] 1,+\infty[)}\right)\left(\varepsilon^{1 / 2}\left\|\widehat{p}_{+, e}^{\mathrm{rem}}\right\|_{\mathrm{L}^{2}(] 1,+\infty[)}\right) \\
& \leq \frac{1}{4 \varepsilon}\left\|\widehat{R}_{+, e}\right\|_{\mathrm{L}^{2}(] 1,+\infty[)}^{2}+\varepsilon\left\|\widehat{p}_{+, e}^{\mathrm{rem}}\right\|_{\mathrm{L}^{2}(] 1,+\infty[)}^{2} \\
& \leq \mathcal{O}\left(\varepsilon^{4}\right)+\varepsilon\left\|\widehat{p}_{+, e}^{\mathrm{rem}}\right\|_{\mathrm{L}^{2}(] 1,+\infty[)}^{2} .
\end{aligned}
$$

Bound for $\left|\int_{-\infty}^{0} \widehat{R}_{-} \widetilde{p}_{-}^{\text {rem }}\right|$.

By a direct computation of the integral we have on the one hand

$$
\left\|\exp \left(\frac{\sigma}{2 \mu \varepsilon}\right)\right\|_{L^{2}(]-\infty, 0[)}=\mathcal{O}\left(\varepsilon^{1 / 2}\right)
$$

and on the other hand

$$
\left\|h(\sigma) \exp \left(\frac{\sigma}{2 \mu \varepsilon}\right)\right\|_{L^{2}(]-\infty, 0[)}=\mathcal{O}\left(\exp \left(\frac{-1}{\mu \varepsilon}\right)\right) .
$$

From Lemma ?? we have

$$
\frac{\Gamma-1}{\mu \varepsilon}=\mathcal{O}(1)
$$

and these three equalities give us

$$
\left\|\widehat{R}_{-}\right\|_{L^{2}(]-\infty, 0[)}=\mathcal{O}\left(\varepsilon^{1 / 2}\right) .
$$

Again, this $\mathrm{L}^{2}$ control and Hölder and Young inequalities give:

$$
\begin{aligned}
\int_{-\infty}^{0} \widehat{R}_{-} \widetilde{p}_{-}^{\mathrm{rem}} & \leq\left\|\widehat{R}_{-}\right\|_{\mathrm{L}^{2}(]-\infty, 0[)}\left(\int_{-\infty}^{0}\left(\widetilde{p}_{-}^{\mathrm{rem}}\right)^{2}\right)^{1 / 2} \\
& \leq\left(\varepsilon\left\|\widehat{R}_{-}\right\|_{\mathrm{L}^{2}(]-\infty, 0[)}\right) \frac{1}{\varepsilon}\left(\int_{-\infty}^{0}\left(\widetilde{p}_{-}^{\mathrm{rem}}\right)^{2}\right)^{1 / 2} \\
& \leq \mathcal{O}\left(\varepsilon^{3}\right)+\frac{1}{8 \mu \varepsilon^{2}} \int_{-\infty}^{0}\left(\widetilde{p}_{-}^{\mathrm{rem}}\right)^{2}
\end{aligned}
$$

Bound for $\mu \Gamma \varepsilon^{2}\left|\widetilde{p}_{+, e}^{\text {rem }}(1)\right|$.

By $(? ?), \mu \Gamma \varepsilon^{2}=\mathcal{O}\left(\varepsilon^{2}\right)$, so all we need to do is bound from above $\left|\widetilde{p}_{+, e}^{\mathrm{rem}}(1)\right|$. To achieve this, we of course use the continuous embedding of $\mathrm{H}^{1}(] 1,+\infty[)$ into 
$\mathrm{L}^{\infty}(] 1,+\infty[)$ and note $C_{\infty}$ the constant of this embedding. We then use Young inequality to get

$$
\begin{aligned}
\mu \Gamma \varepsilon^{2}\left|\widetilde{p}_{+, e}^{\mathrm{rem}}(1)\right| & \leq \mu \Gamma \varepsilon^{3 / 2} C_{\infty} \varepsilon^{1 / 2}\left(\int_{1}^{+\infty}\left(\partial_{\sigma} \widetilde{p}_{+, e}^{\mathrm{rem}}\right)^{2}+\int_{1}^{+\infty}\left(\widetilde{p}_{+, e}^{\mathrm{rem}}\right)^{2}\right)^{1 / 2} \\
& \leq \mu \Gamma\left(\varepsilon^{3} C_{\infty}^{2}+\varepsilon\left(\int_{1}^{+\infty}\left(\partial_{\sigma} \widetilde{p}_{+, e}^{\mathrm{rem}}\right)^{2}+\int_{1}^{+\infty}\left(\widetilde{p}_{+, e}^{\mathrm{rem}}\right)^{2}\right)\right) \\
& \leq \mathcal{O}\left(\varepsilon^{3}\right)+\mu \Gamma \varepsilon \int_{1}^{+\infty}\left(\partial_{\sigma} \widetilde{p}_{+, e}^{\mathrm{rem}}\right)^{2}+\mu \Gamma \varepsilon \int_{1}^{+\infty}\left(\widetilde{p}_{+, e}^{\mathrm{rem}}\right)^{2}
\end{aligned}
$$

Final Form of the Energy Inequality (??).

Since we have $\Gamma \leq 1$ by (??) and $h$ nonnegative, we have

$$
h+\frac{1}{2 \mu \varepsilon^{2}}\left(1-\frac{\Gamma}{2}\right) \geq \frac{1}{4 \mu \varepsilon^{2}} .
$$

Also, by Lemma ?? we have that $\Gamma \rightarrow 1$ when $\varepsilon \rightarrow 0$ so that we may assume $\Gamma \geq 1 / 2$ for $\varepsilon$ small enough. With that we can bound from below the left-hand side of (??)

$$
\begin{gathered}
\mu \Gamma\left(\int_{-\infty}^{0}\left(\partial_{\sigma} \widetilde{p}_{-}^{\mathrm{rem}}\right)^{2}+\int_{0}^{1}\left(\partial_{\sigma} p_{+, i}^{\mathrm{rem}}\right)^{2}+\int_{1}^{+\infty}\left(\partial_{\sigma} \widetilde{p}_{+, e}^{\mathrm{rem}}\right)^{2}\right) \\
+\int_{-\infty}^{0}\left(h+\frac{1}{2 \mu \varepsilon^{2}}\left(1-\frac{\Gamma}{2}\right)\right)\left(\widetilde{p}_{-}^{\mathrm{rem}}\right)^{2}+\int_{1}^{+\infty}\left(\frac{1}{2}-\frac{\mu \Gamma \varepsilon^{2}}{4}\right)\left(\widetilde{p}_{+, e}^{\mathrm{rem}}\right)^{2} \\
\geq \frac{\mu}{2}\left(\int_{-\infty}^{0}\left(\partial_{\sigma} \widetilde{p}_{-}^{\mathrm{rem}}\right)^{2}+\int_{0}^{1}\left(\partial_{\sigma} p_{+, i}^{\mathrm{rem}}\right)^{2}+\int_{1}^{+\infty}\left(\partial_{\sigma} \widetilde{p}_{+, e}^{\mathrm{rem}}\right)^{2}\right) \\
\quad+\frac{1}{4 \mu \varepsilon^{2}} \int_{-\infty}^{0}\left(\widetilde{p}_{-}^{\mathrm{rem}}\right)^{2}+\int_{1}^{+\infty}\left(\frac{1}{2}-\frac{\mu \varepsilon^{2}}{4}\right)\left(\widetilde{p}_{+, e}^{\mathrm{rem}}\right)^{2} .
\end{gathered}
$$

We now bound from above the three terms of the right-hand side of (??) using (??)-(??) to obtain that

$$
\begin{aligned}
& \mu \Gamma \varepsilon^{2}\left|\widetilde{p}_{+, e}^{\mathrm{rem}}(1)\right|+\left|\int_{-\infty}^{0} \widehat{R}_{-} \widetilde{p}_{-}^{\mathrm{rem}}\right|+\left|\int_{1}^{+\infty} \widehat{R}_{+, e} \widetilde{p}_{+, e}^{\mathrm{rem}}\right| \\
& \leq \mathcal{O}\left(\varepsilon^{3}\right)+\frac{1}{8 \mu \varepsilon^{2}} \int_{-\infty}^{0}\left(\widetilde{p}_{-}^{\mathrm{rem}}\right)^{2}+\mu \varepsilon \int_{1}^{+\infty}\left(\partial_{\sigma} \widetilde{p}_{+, e}^{\mathrm{rem}}\right)^{2}+(\mu+1) \varepsilon \int_{1}^{+\infty}\left(\widetilde{p}_{+, e}^{\mathrm{rem}}\right)^{2}
\end{aligned}
$$

We can now write the final form of the energy inequality:

$$
\begin{aligned}
& \mu\left(\frac{1}{2}-\varepsilon\right) \int_{1}^{+\infty}\left(\partial_{\sigma} \widetilde{p}_{+, e}^{\mathrm{rem}}\right)^{2}+\left(\frac{1}{2}-\frac{\mu \varepsilon^{2}}{4}-(\mu+1) \varepsilon\right) \int_{1}^{+\infty}\left(\widetilde{p}_{+, e}^{\mathrm{rem}}\right)^{2} \\
& \quad+\frac{\mu}{2} \int_{-\infty}^{0}\left(\partial_{\sigma} \widetilde{p}_{-}^{\mathrm{rem}}\right)^{2}+\frac{1}{8 \mu \varepsilon^{2}} \int_{-\infty}^{0}\left(p_{-}^{\mathrm{rem}}\right)^{2}+\frac{\mu}{2} \int_{0}^{1}\left(\partial_{\sigma} p_{+, i}^{\mathrm{rem}}\right)^{2} \leq \mathcal{O}\left(\varepsilon^{3}\right) .
\end{aligned}
$$


Proof of Inequalities (??)-(??).

We can extract from (??) the following estimates:

$$
\begin{aligned}
\left\|\widetilde{p}_{-}^{\mathrm{rem}}\right\|_{\mathrm{L}^{2}(]-\infty, 0[)} & =\mathcal{O}\left(\varepsilon^{5 / 2}\right), \\
\left\|\widetilde{p}_{+, e}^{\mathrm{rem}}\right\|_{L^{2}(1,+\infty} & =\mathcal{O}\left(\varepsilon^{3 / 2}\right),
\end{aligned}
$$

which are exactly (??) and (??). To complete the proof of Proposition ?? we need to show the estimate (??). Since we do not get it directly from (??) we have to use some kind of Poincaré inequality. For this we write, for $\sigma \in[0,1]$ :

$$
\left(p_{+, i}^{\mathrm{rem}}\right)^{2}(\sigma)=\int_{0}^{\sigma} 2 \partial_{\sigma} p_{+, i}^{\mathrm{rem}}(s) p_{+, i}^{\mathrm{rem}}(s) \mathrm{d} s+\left(p_{+, i}^{\mathrm{rem}}(0)\right)^{2} .
$$

We can now use the transmission condition of (??) to write:

$$
\begin{aligned}
\left(p_{+, i}^{\mathrm{rem}}\right)^{2}(\sigma) & =\int_{0}^{\sigma} 2 \sqrt{2} \partial_{\sigma} p_{+, i}^{\mathrm{rem}}(s) \frac{1}{\sqrt{2}} p_{+, i}^{\mathrm{rem}}(s) \mathrm{d} s+\left(p_{-}^{\mathrm{rem}}(0)\right)^{2} \\
& \leq 2 \int_{0}^{\sigma}\left(\partial_{\sigma} p_{+, i}^{\mathrm{rem}}(s)\right)^{2} \mathrm{~d} s+\frac{1}{2} \int_{0}^{\sigma}\left(p_{+, i}^{\mathrm{rem}}(s)\right)^{2} \mathrm{~d} s+\left(\int_{-\infty}^{0} \partial_{\sigma} p_{-}^{\mathrm{rem}}\right)^{2} \\
& \leq 2 \int_{0}^{1}\left(\partial_{\sigma} p_{+, i}^{\mathrm{rem}}\right)^{2}+\frac{1}{2} \int_{0}^{1}\left(p_{+, i}^{\mathrm{rem}}\right)^{2}+\left(\int_{-\infty}^{0} \partial_{\sigma} p_{-}^{\mathrm{rem}}\right)^{2} .
\end{aligned}
$$

We now integrate this inequality between 0 and 1 to obtain:

$$
\int_{0}^{1}\left(p_{+, i}^{\mathrm{rem}}\right)^{2} \leq 4 \int_{0}^{1}\left(\partial_{\sigma} p_{+, i}^{\mathrm{rem}}\right)^{2}+2\left(\int_{-\infty}^{0} \partial_{\sigma} p_{-}^{\mathrm{rem}}\right)^{2} .
$$

Now from (??) we already have:

$$
\int_{0}^{1}\left(\partial_{\sigma} p_{+, i}^{\mathrm{rem}}\right)^{2}=\mathcal{O}\left(\varepsilon^{3}\right)
$$

Moreover, we have seen that

$$
\partial_{\sigma} p_{-}^{\mathrm{rem}}=\exp \left(\frac{\sigma}{2 \mu \varepsilon}\right)\left(\partial_{\sigma} \widetilde{p}_{-}^{\mathrm{rem}}(\sigma)+\frac{1}{2 \mu \varepsilon} \widetilde{p}_{-}^{\mathrm{rem}}(\sigma)\right) .
$$

and by (??) we have both

$$
\begin{gathered}
\left\|\frac{1}{2 \mu \varepsilon} \widehat{p}_{-}^{\mathrm{rem}}\right\|_{L^{2}(]-\infty, 0[)}=\mathcal{O}\left(\varepsilon^{3 / 2}\right), \\
\left\|\partial_{\sigma} \widetilde{p}_{-}^{\mathrm{rem}}\right\|_{L^{2}(]-\infty, 0[)}=\mathcal{O}\left(\varepsilon^{3 / 2}\right),
\end{gathered}
$$

so that by Hölder inequality,

$$
\begin{aligned}
\int_{-\infty}^{0} \partial_{\sigma} p_{-}^{\mathrm{rem}} & =\int_{-\infty}^{0} \exp \left(\frac{\sigma}{2 \mu \varepsilon}\right)\left(\partial_{\sigma} \widehat{p}_{-}^{\mathrm{rem}}(\sigma)+\frac{1}{2 \mu \varepsilon} \widehat{p}_{-}^{\mathrm{rem}}(\sigma)\right) \mathrm{d} \sigma \\
& \left.\leq\left(\int_{-\infty}^{0} \exp \left(\frac{\sigma}{\mu \varepsilon}\right)\right)^{1 / 2}\left\|\partial_{\sigma} \widetilde{p}_{-}^{\mathrm{rem}}+\frac{1}{2 \mu \varepsilon} \widetilde{p}_{-}^{\mathrm{rem}}\right\|_{L^{2}(]-\infty, 0[)}\right) \\
& \leq(\mu \varepsilon)^{1 / 2}\left(\left\|\partial_{\sigma} \widetilde{p}_{-}^{\mathrm{rem}}\right\|_{L^{2}(]-\infty, 0[)}+\left\|\frac{1}{2 \mu \varepsilon} \widetilde{p}_{-}^{\mathrm{rem}}\right\|_{L^{2}(]-\infty, 0[)}\right) .
\end{aligned}
$$


Consequently,

$$
\left(\int_{-\infty}^{0} \partial_{\sigma} p_{-}^{\mathrm{rem}}\right)^{2}=\mathcal{O}\left(\varepsilon^{4}\right)
$$

which gives

$$
\int_{0}^{1}\left(p_{+, i}^{\mathrm{rem}}\right)^{2}=\mathcal{O}\left(\varepsilon^{3}\right),
$$

that is (??). We have thus proved Proposition ??.

Remark 1. On a side note, the two equalities,

$$
\begin{aligned}
& \partial_{\sigma} p_{-}^{\mathrm{rem}}(\sigma)=\exp \left(\frac{\sigma}{2 \mu \varepsilon}\right)\left(\partial_{\sigma} \widetilde{p}_{-}^{\mathrm{rem}}(\sigma)+\frac{1}{2 \mu \varepsilon} \widetilde{p}_{-}^{\mathrm{rem}}(\sigma)\right), \\
& \partial_{\sigma} p_{+, e}^{\mathrm{rem}}(\sigma)=\exp \left(-\frac{\varepsilon}{2}(\sigma-1)\right)\left(\partial_{\sigma} \widetilde{p}_{+, e}^{\mathrm{rem}}(\sigma)-\frac{\varepsilon}{2} \widetilde{p}_{+, e}^{\mathrm{rem}}(\sigma)\right),
\end{aligned}
$$

the energy estimate (??) and (??) prove that we also have the estimate

$$
\left\|p-p^{\mathrm{app}}\right\|_{\mathrm{H}^{1}(\mathbf{R})}=\mathcal{O}\left(\varepsilon^{3 / 2}\right) .
$$

\section{Proof of theo ??}

In this section, we deduce theo ?? from Proposition ??, which was proved in the previous section.

Let us note first that by a direct computation we can show that:

$$
\int_{\sigma \in \mathbf{R}} \sigma p^{\operatorname{app}}(\sigma) \mathrm{d} \sigma \sim \frac{1}{\varepsilon} .
$$

All that is left to prove is that the stress attached to the remainder is o $(1 / \varepsilon)$.

We use the symbol $A \lesssim B$ to express that there is a positive constant $C$ independent of $\varepsilon$, such that

$$
A \leq C \cdot B .
$$

Since we have a weighted control of the norm of $p_{-}^{\text {rem }}$ and $p_{+, e}^{\text {rem }}$, we can use the following inequalities, which are true for small $\varepsilon$ :

$$
\begin{aligned}
\left|\int_{-\infty}^{0} \sigma p_{-}^{\mathrm{rem}}(\sigma) \mathrm{d} \sigma\right| & \leq\left(\int_{-\infty}^{0} \sigma^{2} \exp \left(\frac{\sigma}{\mu \varepsilon}\right) \mathrm{d} \sigma\right)^{1 / 2}\left\|\widehat{p}_{-}^{\mathrm{rem}}\right\|_{\mathrm{L}^{2}(]-\infty, 0[)} \\
& \lesssim(\mu \varepsilon)^{3 / 2}\left\|\widehat{p}_{-}^{\mathrm{rem}}\right\|_{L^{2}(]-\infty, 0[)} \\
\left|\int_{1}^{+\infty} \sigma p_{+, e}^{\mathrm{rem}}(\sigma) \mathrm{d} \sigma\right| & \leq\left(\int_{1}^{+\infty} \sigma^{2} \exp (-\varepsilon(\sigma-1)) \mathrm{d} \sigma\right)^{1 / 2}\left\|\widetilde{p}_{-}^{\mathrm{rem}}\right\|_{L^{2}(] 1,+\infty[)} \\
& \lesssim \frac{1}{\varepsilon^{3 / 2}}\left\|\widehat{p}_{-}^{\mathrm{rem}}\right\|_{\mathrm{L}^{2}(] 1,+\infty[)}
\end{aligned}
$$

We also have

$$
\begin{aligned}
\left|\int_{0}^{1} \sigma p_{+, i}^{\mathrm{rem}}(\sigma) \mathrm{d} \sigma\right| & \leq\left(\int_{0}^{1} \sigma^{2}\right)^{1 / 2}\left\|p_{+, i}^{\mathrm{rem}}\right\|_{\mathrm{L}^{2}(] 0,1[)} \\
& \lesssim\left\|p_{+, i}^{\mathrm{rem}}\right\|_{\left.\left.\mathrm{L}^{2}(] 0,1\right]\right)}
\end{aligned}
$$


Then it suffices to combine inequalities (??) with (??), (??) with (??) and (??) with (??) to obtain:

$$
\left|\int_{\sigma \in \mathbf{R}} \sigma p^{\mathrm{rem}}(\sigma) \mathrm{d} \sigma\right| \leq \mathcal{O}(1)
$$

so that we have the expansion

$$
\tau=\frac{1}{\varepsilon}+\mathcal{O}(1)
$$

and theo ??

\section{Proof of Lemma??}

This section is devoted to the proof of Lemma ??. What's interesting is that the proof of this lemma requires the study of a singular limit by itself. Indeed to gain an estimate on $\Gamma$ we need to approximate the remainder problem (??) by a linear approximate remainder problem (??). Contrary to what happens in the small shear rate limit we carried out in [?] the expansion of $p$ and $\tau$ are uniform in $\mu$ and, as such, much simpler. The difficulty of the large shear rate limit, however, is that there is no real limit in this problem. Indeed, a naïve approach would be to say that since the singular term is $1 / \varepsilon \partial_{\sigma} p$ then, $p$ should go toward a constant function and this constant can only be 0 . This is incompatible with the fact that "in the limit" the integral should still be one.

Let us first introduce the approximate remainder problem and explain its link to System (??):

we define the functions $\pi_{-}^{C, \varepsilon}, \pi_{+, i}^{C, \varepsilon}$ and $\pi_{+, e}^{C, \varepsilon}$ to be the solutions of the following system,

$$
\left\{\begin{array}{l}
-\mu(1+C \varepsilon) \partial_{\sigma}^{2} \pi_{-}^{C, \varepsilon}+h \pi_{-}^{C, \varepsilon}+\frac{1}{\varepsilon} \partial_{\sigma} \pi_{-}^{C, \varepsilon}=\bar{R}_{-} \\
-\mu(1+C \varepsilon) \partial_{\sigma}^{2} \pi_{+, i}^{C, \varepsilon}+\frac{1}{\varepsilon} \partial_{\sigma} \pi_{+, i}^{C, \varepsilon}=0 \\
-\mu(1+C \varepsilon) \partial_{\sigma}^{2} \pi_{+, e}^{C, \varepsilon}+\pi_{+, e}^{C, \varepsilon}+\frac{1}{\varepsilon} \partial_{\sigma} \pi_{+, e}^{C, \varepsilon}=\bar{R}_{+, e} \\
\pi_{+, i}^{C, \varepsilon}(0)=\pi_{-}^{C, \varepsilon}(0) \\
\pi_{+, e}^{C, \varepsilon}(1)=\pi_{+, i}^{C, \varepsilon}(1) \\
\partial_{\sigma} \pi_{+, i}^{C, \varepsilon}(0)=\partial_{\sigma} \pi_{-}^{C, \varepsilon}(0) \\
\partial_{\sigma} \pi_{+, e}^{C, \varepsilon}(1)-\varepsilon^{2}=\partial_{\sigma} \pi_{+, i}^{C, \varepsilon}(1)
\end{array}\right.
$$

where

$$
\begin{aligned}
\bar{R}_{-} & =\left(\frac{C}{\mu}-\varepsilon h\right) \exp \left(\frac{\sigma}{\mu \varepsilon}\right), \\
\bar{R}_{+, e} & =\mu(1+C \varepsilon) \varepsilon^{3} \exp (-\varepsilon(\sigma-1)) .
\end{aligned}
$$

We also define the two variable function

$$
F(C, \varepsilon)=\int_{-\infty}^{0} \pi_{-}^{C, \varepsilon}+\int_{0}^{1} \pi_{+, i}^{C, \varepsilon}+\int_{1}^{+\infty} \pi_{+, i}^{C, \varepsilon}+\varepsilon-\mu \varepsilon^{2} .
$$


Replace, in (??), $C$ by $\Gamma^{\mathrm{rem}}$ and the system becomes (??) without the integral constraint. Thus the solution to (??) with $C=\Gamma^{\mathrm{rem}}$, which is by definition $\pi^{\Gamma^{\mathrm{rem}}, \varepsilon}$ is also the solution to (??) $p^{\mathrm{rem}}$. Consequently the integral constraint of (??) is exactly the equation $F\left(\Gamma^{\mathrm{rem}}, \varepsilon\right)=0$. Conversely, for any $C$ verifying $F(C, \varepsilon)=0$ we have that $\pi^{C, \varepsilon}$ satisfies completely the problem (??), including the integral constraint and thus we have $C=\Gamma^{\mathrm{rem}}$. This argument relies on the unique solvability of (??) for fixed $\varepsilon$ or, equivalently, on the unique solvability of (??). This property has already been proved in [?] or [?].

Let us first state that we have the analogous to Proposition ?? for (??). Since there will be a boundary layer on the left side of $\sigma=1$ we introduce $\theta$, a $\mathcal{C}^{\infty}$ localization function which is 1 on $[1 / 2,1], 0$ on $[0,1 / 3]$ and strictly increasing on $] 1 / 3,1 / 2[$. Then we have the following approximation result:

Proposition 2. The solution to the remainder problem (??) noted by $\left(\pi_{-}^{C, \varepsilon}, \pi_{+, i}^{C, \varepsilon}, \pi_{+, e}^{C, \varepsilon}\right)$ can be expanded, when $\varepsilon \rightarrow 0$, as $\pi^{C, \varepsilon}=\pi^{C, \varepsilon, \text { app }}+\pi^{C, \varepsilon, \text { rem }}$ where:

$$
\begin{aligned}
& \pi_{-}^{C, \varepsilon, \operatorname{app}}(\sigma)=C \varepsilon^{2}\left(1-\frac{C \sigma}{\mu \varepsilon}\right) \exp \left(\frac{\sigma}{\varepsilon}\right), \\
& \pi_{+, i}^{C, \varepsilon, \operatorname{app}}(\sigma)=C \varepsilon^{2}+\mu \varepsilon^{3} \theta(\sigma)\left(1-\exp \left(\frac{\sigma-1}{\mu \varepsilon}\right)\right), \\
& \pi_{+, e}^{C, \varepsilon, \operatorname{app}}(\sigma)=C \varepsilon^{2} \exp (-\varepsilon(\sigma-1)),
\end{aligned}
$$

with the following weighted $\mathrm{L}^{2}$ controls:

$$
\begin{aligned}
\left\|\exp \left(-\frac{\cdot}{2 \mu \varepsilon}\right) \pi_{-}^{C, \varepsilon, \text { rem }}\right\|_{L^{2}(]-\infty, 0[)} & =\mathcal{O}\left(\varepsilon^{7 / 2}\right), \\
\left\|\pi_{+, i}^{C, \varepsilon, \text { rem }}\right\|_{L^{2}(] 0,1[)} & =\mathcal{O}\left(\varepsilon^{5 / 2}\right), \\
\left\|\exp \left(\frac{\varepsilon}{2}(\cdot-1)\right) \pi_{+, e}^{C, \varepsilon, \text { rem }}\right\|_{L^{2}(1, \infty)} & =\mathcal{O}\left(\varepsilon^{5 / 2}\right) .
\end{aligned}
$$

We will prove this proposition in Section ??. From this proposition we can deduce the following result which will be detailed in Section ??.

Proposition 3. For a fixed $C$ we have:

$$
F(C, \varepsilon)=(C+1) \varepsilon+\mathcal{O}\left(\varepsilon^{2}\right) .
$$

Because of the linear and elliptic nature of (??) it is clear that $F$ must be at least continuous in $C, \varepsilon$ in the domain $1+C \varepsilon>0$. Let us fix two constant numbers $C_{1}$ and $C_{2}$ such that $C_{1}<-1<C_{2}$. Then because of Proposition ?? and because $F$ is continuous there is an interval $] 0, \varepsilon_{0}$ [ on which we have

$$
\forall \varepsilon \in] 0, \varepsilon_{0}\left[, \quad F\left(C_{1}, \varepsilon\right)<0<F\left(C_{2}, \varepsilon\right) .\right.
$$

Now for any fixed $\varepsilon \in] 0, \varepsilon_{0}$ [ we use the continuity in $C$ of $F$ and the intermediate value theo to affirm that there must be a 0 of $F(\cdot, \varepsilon)$ on the interval $\left[C_{1}, C_{2}\right]$. But as we pointed out, the only 0 that $F(\cdot, \varepsilon)$ has is $\Gamma^{\mathrm{rem}}$. Consequently,

$$
\forall \varepsilon \in] 0, \varepsilon^{0}\left[, \quad C_{1}<\Gamma^{\mathrm{rem}}<C_{2},\right.
$$

which is exactly what Lemma ?? states. 
Remark 2. Note that $C=-1$ makes $F(C, \varepsilon)$ vanish for $\varepsilon \rightarrow 0$ faster than any other value of $C$ and indeed one could prove by expanding $p$ at a higher order that we formally have $\Gamma=1-\varepsilon+\mathcal{O}\left(\varepsilon^{2}\right)$ and also that $p^{\text {app }}+\pi^{-1, \varepsilon \text {,app }}$ is the second order expansion of $p$.

\subsection{Proof of Proposition ??}

This section is devoted to construct an approximation to System (??) and to estimate the remainder of this approximation. It is very similar to Sections ?? and ??. Consequently, we will not give as much details.

To find the asymptotic behaviour of $\pi^{C, \varepsilon}$ we once again change variables and set, now classically:

$$
\begin{array}{ll}
\pi_{-}^{C, \varepsilon}(\sigma)=\chi_{-}\left(\frac{\sigma}{\varepsilon}\right), & \pi_{+, i}^{C, \varepsilon}(\sigma)=\chi_{+, i}(\sigma)+\chi_{b l}\left(\frac{1-\sigma}{\varepsilon}\right), \\
\pi_{+, e}^{C, \varepsilon}(\sigma) & =\chi_{+, e}(\varepsilon(\sigma-1)),
\end{array}
$$

so that the functions $\chi_{-}, \chi_{+, i}, \chi_{b l}$ and $\chi_{+, e}$ satisfy the following problem:

$$
\left\{\begin{array}{l}
-\mu(1+C \varepsilon) \partial_{z}^{2} \chi_{-}+\varepsilon^{2} h_{\varepsilon} \chi_{-}+\partial_{z} \chi_{-}=\varepsilon^{2}\left(\frac{C}{\mu}-\varepsilon h_{\varepsilon}\right) \exp \left(\frac{z}{\mu}\right) \\
-\mu(1+C \varepsilon) \partial_{\sigma}^{2} \chi_{+, i}+\frac{1}{\varepsilon} \partial_{\sigma} \chi_{+, i}=0 \\
-\mu(1+C \varepsilon) \partial_{z}^{2} \chi_{b l}-\partial_{z} \chi_{b l}=0 \\
-\mu \varepsilon^{2}(1+C \varepsilon) \partial_{z}^{2} \chi_{+, e}+\chi_{+, e}+\partial_{z} \chi_{+, e}=\mu(1+C \varepsilon) \varepsilon^{3} \exp (-z) \\
\chi_{+, i}(0)+\chi_{b l}\left(\frac{1}{\varepsilon}\right)=\chi_{-}(0) \\
\chi_{+, e}(0)=\chi_{+, i}(1) \\
\partial_{\sigma} \chi_{+, i}(0)=\frac{1}{\varepsilon} \partial_{z} \chi_{-}(0) \\
\varepsilon \partial_{z} \chi_{+, e}(0)-\varepsilon^{2}=\partial_{\sigma} \chi_{+, i}(1)-\frac{1}{\varepsilon} \partial_{z} \chi_{b l}
\end{array}\right.
$$

and assume an expansion of the form

$$
\begin{aligned}
\chi_{-} & =\varepsilon^{2} \chi_{-}^{1}+\varepsilon^{2} \chi_{-}^{2}+\varepsilon^{3} \chi_{-}^{3}+\ldots, \\
\chi_{+, i} & =\varepsilon^{2} \chi_{+, i}^{1}+\varepsilon^{2} \chi_{+, i}^{2}+\varepsilon^{3} \chi_{+, i}^{3}+\ldots, \\
\chi_{b l} & =\varepsilon^{3} \chi_{b l}^{3}+\varepsilon^{4} \chi_{b l}^{4}+\ldots, \\
\chi_{+, e} & =\varepsilon^{2} \chi_{+, e}^{1}+\varepsilon^{2} \chi_{+, e}^{2}+\varepsilon^{3} \chi_{+, e}^{3} \ldots
\end{aligned}
$$

Recall that, to obtain, (??) we used a truncation at order $\varepsilon$ of the formal expansion of $p$, so that $p^{\text {rem }}$ is formally of order $\varepsilon^{2}$. Now $\pi^{\text {rem, } C, \varepsilon}$ solves (??), a problem which is very similar to (??), and which is hopefully at the same formal $\varepsilon$ order. It is thus natural to see that the first order of $\pi^{\mathrm{rem}, C, \varepsilon}$ should be $\varepsilon^{2}$. The following analysis will prove that the previous formal expansions give the correct ansaetze.

One interesting point here is that when solving the profile equations for (??), we start with $\sigma>1$ because the most singular term comes from equation 


$$
\varepsilon \int_{-\infty}^{0} q_{-}(z) \mathrm{d} z+\int_{0}^{1} q_{+, i}(z) \mathrm{d} z+\varepsilon \int_{0}^{1 / \varepsilon} q_{b l}(z) \mathrm{d} z+\frac{1}{\varepsilon} \int_{0}^{+\infty} q_{+, e}(z) \mathrm{d} z=1 .
$$

But such a constraint has disappeared in (??) and the most singular term now comes from

$$
\partial_{\sigma} \chi_{+, i}(0)=\frac{1}{\varepsilon} \partial_{z} \chi_{-}(0)
$$

and we solve from left $(\sigma<0)$ to right $(\sigma>1)$. It is then not difficult to see that the lowest order approximation of $\pi^{C, \varepsilon}$ is formally given by

$$
\begin{aligned}
& \pi_{-}^{C, \varepsilon, \operatorname{app}}(\sigma)=C \varepsilon^{2}\left(1-\frac{C \sigma}{\mu \varepsilon}\right) \exp \left(\frac{\sigma}{\varepsilon}\right) \\
& \pi_{+, i}^{C, \varepsilon, \operatorname{app}}(\sigma)=C \varepsilon^{2}+\mu \varepsilon^{3} \theta(\sigma)\left(1-\exp \left(\frac{\sigma-1}{\mu \varepsilon}\right)\right) \\
& \pi_{+, e}^{C, \varepsilon, \operatorname{app}}(\sigma)=C \varepsilon^{2} \exp (-\varepsilon(\sigma-1)) .
\end{aligned}
$$

These expressions should be compared to the second order profiles given in Section ??. We now again set $\pi^{C, \varepsilon, \text { rem }}=\pi^{C, \varepsilon}-\pi^{C, \varepsilon, \text { app }}$. Then $\pi_{-}^{C, \varepsilon, \text { rem }}, \pi_{+, i}^{C, \varepsilon, \text { rem }}$ and $\pi_{+, e}^{C, \varepsilon, \text { rem }}$ solve the following problem:

$$
\left\{\begin{array}{l}
-\mu(1+C \varepsilon) \partial_{\sigma}^{2} \pi_{-}^{C, \varepsilon, \mathrm{rem}}+h \pi_{-}^{C, \varepsilon, \mathrm{rem}}+\frac{1}{\varepsilon} \partial_{\sigma} \pi_{-}^{C, \varepsilon, \mathrm{rem}}=\widetilde{R}_{-}, \\
-\mu(1+C \varepsilon) \partial_{\sigma}^{2} \pi_{+, i}^{C, \varepsilon, \mathrm{rem}}+\frac{1}{\varepsilon} \partial_{\sigma} \pi_{+, i}^{C, \varepsilon, \mathrm{rem}}=\widetilde{R}_{+, i}, \\
-\mu(1+C \varepsilon) \partial_{\sigma}^{2} \pi_{+, e}^{C, \varepsilon, \mathrm{rem}}+\pi_{+, e}^{C, \varepsilon, \mathrm{rem}}+\frac{1}{\varepsilon} \partial_{\sigma} \pi_{+, e}^{C, \varepsilon, \mathrm{rem}}=\widetilde{R}_{+, e}, \\
\pi_{+, i}^{C, \varepsilon, \mathrm{rem}}(0)=\pi_{-}^{C, \varepsilon, \mathrm{rem}}(0), \\
\pi_{+, e}^{C, \varepsilon, \mathrm{rem}}(1)=\pi_{+, i}^{C, \varepsilon, \mathrm{rem}}(1), \\
\partial_{\sigma} \pi_{+, i}^{C, \varepsilon, \mathrm{rem}}(0)=\partial_{\sigma} \pi_{-}^{C, \varepsilon, \mathrm{rem}}(0), \\
\partial_{\sigma} \pi_{+, e}^{C, \varepsilon, \mathrm{rem}}(1)=\partial_{\sigma} \pi_{+, i}^{C, \varepsilon, \mathrm{rem}}(1),
\end{array}\right.
$$

where

$$
\begin{aligned}
\widetilde{R}_{-} & =-C\left(\frac{\sigma}{\mu^{2}}+\frac{C \varepsilon}{\mu}\right) \exp \left(\frac{\sigma}{2 \mu \varepsilon}\right)-\left(C \varepsilon^{2}\left(1-\frac{\sigma}{\mu \varepsilon}\right)+\varepsilon\right) h \exp \left(\frac{\sigma}{2 \mu \varepsilon}\right), \\
\widetilde{R}_{+, i} & =-\mu \varepsilon^{2} \theta^{\prime}+\mu^{2} \varepsilon^{3} \theta^{\prime \prime}+3 \mu \varepsilon^{2} \theta^{\prime} \exp \left(\frac{\sigma-1}{\mu \varepsilon}\right)-\mu^{2} \varepsilon^{3} \theta^{\prime \prime} \exp \left(\frac{\sigma-1}{\mu \varepsilon}\right), \\
\widetilde{R}_{+, e} & =\mu \varepsilon^{3}(1+C \varepsilon) \exp \left(-\frac{\varepsilon}{2}(\sigma-1)\right)+C \varepsilon^{4}(1+C \varepsilon) \exp \left(-\frac{\varepsilon}{2}(\sigma-1)\right) .
\end{aligned}
$$

Let us again introduce

$$
\begin{aligned}
& \pi_{-}^{C, \varepsilon, \mathrm{rem}}=\exp \left(\frac{\sigma}{2 \mu \varepsilon}\right) \tilde{\pi}_{-}^{C, \varepsilon, \mathrm{rem}} \\
& \pi_{+, e}^{C, \varepsilon, \mathrm{rem}}=\exp \left(-\frac{\varepsilon}{2}(\sigma-1)\right) \widetilde{\pi}_{+, e}^{C, \varepsilon, \mathrm{rem}}
\end{aligned}
$$


We are going to prove that $\tilde{\pi}_{-}^{C, \varepsilon, \text { rem }}$ and $\tilde{\pi}_{+, e}^{C, \varepsilon, \text { rem }}$, are in $\mathrm{L}^{2}(]-\infty, 0[)$ and $\mathrm{L}^{2}(] 1,+\infty[)$ respectively. Let us write the problem solved by $\tilde{\pi}_{-}^{C, \varepsilon, \mathrm{rem}}, \pi_{+, i}^{C, \varepsilon, \mathrm{rem}}$ and $\tilde{\pi}_{+, e}^{C, \varepsilon, \mathrm{rem}}$ :

$$
\left\{\begin{array}{l}
-\mu(1+C \varepsilon) \partial_{\sigma}^{2} \widetilde{\pi}_{-}^{C, \varepsilon, \mathrm{rem}}+\left(h+\frac{1}{4 \mu \varepsilon^{2}}-\frac{C}{4 \mu \varepsilon}\right) \tilde{\pi}_{-}^{C, \varepsilon, \mathrm{rem}}-\frac{C}{\mu} \partial_{\sigma} \widetilde{\pi}_{-}^{C, \varepsilon, \mathrm{rem}}=\widetilde{R}_{-}, \\
-\mu(1+C \varepsilon) \partial_{\sigma}^{2} \pi_{+, i}^{C, \varepsilon, \mathrm{rem}}+\frac{1}{\varepsilon} \partial_{\sigma} \pi_{+, i}^{C, \varepsilon, \mathrm{rem}}=\widetilde{R}_{+, i}, \\
-\mu(1+C \varepsilon) \partial_{\sigma}^{2} \widetilde{\pi}_{+, e}^{C, \varepsilon, \mathrm{rem}}+\left(\frac{1}{2}-\frac{\mu(1+C \varepsilon) \varepsilon^{2}}{4}\right) \tilde{\pi}_{+, e}^{C, \varepsilon, \mathrm{rem}} \\
\quad+\left(\frac{1}{\varepsilon}-\mu(1+C \varepsilon) \varepsilon^{2}\right) \partial_{\sigma} \widetilde{\pi}_{+, e}^{C, \varepsilon, \mathrm{rem}}=\widetilde{R}_{+, e}, \\
\pi_{+, i}^{C, \varepsilon, \mathrm{rem}}(0)=\widetilde{\pi}_{-}^{C, \varepsilon, \mathrm{rem}}(0), \\
\tilde{\pi}_{+, e}^{C, \varepsilon, \mathrm{rem}}(1)=\pi_{+, i}^{C, \varepsilon, \mathrm{rem}}(1), \\
\partial_{\sigma} \pi_{+, i}^{C, \varepsilon, \mathrm{rem}}(0)=\partial_{\sigma} \widetilde{\pi}_{-}^{C, \varepsilon, \mathrm{rem}}(0)+\frac{1}{2 \mu \varepsilon} \widetilde{\pi}_{-}^{C, \varepsilon, \mathrm{rem}}(0), \\
\partial_{\sigma} \widetilde{\pi}_{+, e}^{C, \varepsilon, \mathrm{rem}}(1)-\frac{\varepsilon}{2} \widetilde{\pi}_{+, e}^{C, \varepsilon, \mathrm{rem}}(1)=\partial_{\sigma} \pi_{+, i}^{C, \varepsilon, \mathrm{rem}}(1) .
\end{array}\right.
$$

Now we can obtain the same kind of energy estimate as (??):

$$
\begin{aligned}
\mu(1+C \varepsilon)\left(\int_{-\infty}^{0}\left(\partial_{\sigma} \widetilde{\pi}_{-}^{C, \varepsilon, \mathrm{rem}}\right)^{2}+\int_{0}^{1}\left(\partial_{\sigma} \pi_{+, i}^{C, \varepsilon, \mathrm{rem}}\right)^{2}+\int_{1}^{+\infty}\left(\partial_{\sigma} \widetilde{\pi}_{+, e}^{C, \varepsilon, \mathrm{rem}}\right)^{2}\right) \\
\quad+\frac{1}{8 \mu \varepsilon^{2}} \int_{-\infty}^{0}\left(\widetilde{\pi}_{-}^{C, \varepsilon, \mathrm{rem}}\right)^{2}+\frac{1}{2} \int_{1}^{+\infty}\left(\widetilde{\pi}_{+, e}^{C, \varepsilon, \mathrm{rem}}\right)^{2} \\
\leq \int_{-\infty}^{0} \widetilde{R}_{-} \widetilde{\pi}_{-}^{C, \varepsilon, \mathrm{rem}} \pi_{+, i}^{C, \varepsilon, \mathrm{rem}}+\int_{0}^{1} \widetilde{R}_{+, i}+\int_{1}^{+\infty} \widetilde{R}_{+, e} \widetilde{\pi}_{+, e}^{C, \varepsilon, \mathrm{rem}}
\end{aligned}
$$

Once again we can absorb the right-hand side into the left hand side by noting that:

$$
\begin{aligned}
& \left\|\widetilde{R}_{-}\right\|_{\mathrm{L}^{2}(]-\infty, 0[)}=\mathcal{O}\left(\varepsilon^{3 / 2}\right), \\
& \left\|\widetilde{R}_{+, e}\right\|_{\mathrm{L}^{2}(1, \infty)}=\mathcal{O}\left(\varepsilon^{5 / 2}\right), \\
& \left\|\widetilde{R}_{+, i}\right\|_{\left.\left.L^{2}(] 0,1\right]\right)}=\mathcal{O}\left(\varepsilon^{2}\right) .
\end{aligned}
$$


Also note that we have the following a priori estimate:

$$
\begin{aligned}
\int_{0}^{1}\left(\pi_{+, i}^{C, \varepsilon, \mathrm{rem}}\right)^{2} \lesssim & \left(\pi_{+, i}^{C, \varepsilon, \mathrm{rem}}(0)\right)^{2}+\int_{0}^{1}\left(\partial_{\sigma} \pi_{+, i}^{C, \varepsilon, \mathrm{rem}}\right)^{2} \\
\lesssim & \left(\int_{-\infty}^{0} \partial_{\sigma} \pi_{-}^{C, \varepsilon, \mathrm{rem}}\right)^{2}+\int_{0}^{1}\left(\partial_{\sigma} \pi_{+, i}^{C, \varepsilon, \mathrm{rem}}\right)^{2} \\
\lesssim & \left(\int_{-\infty}^{0} \partial_{\sigma}\left(\exp \left(\frac{\sigma}{2 \mu \varepsilon}\right) \tilde{\pi}_{-}^{C, \varepsilon, \mathrm{rem}}\right)\right)^{2}+\int_{0}^{1}\left(\partial_{\sigma} \pi_{+, i}^{C, \varepsilon, \mathrm{rem}}\right)^{2} \\
\lesssim & \left(\int_{-\infty}^{0} \exp \left(\frac{\sigma}{2 \mu \varepsilon}\right)\left(\partial_{\sigma} \widetilde{\pi}_{-}^{C, \varepsilon, \mathrm{rem}}+\frac{1}{2 \mu \varepsilon} \widetilde{\pi}_{-}^{C, \varepsilon, \mathrm{rem}}\right)\right)^{2} \\
& +\int_{0}^{1}\left(\partial_{\sigma} \pi_{+, i}^{C, \varepsilon, \mathrm{rem}}\right)^{2} \\
\lesssim & \varepsilon^{1 / 2}\left(\int_{-\infty}^{0}\left(\partial_{\sigma} \widetilde{\pi}_{-}^{C, \varepsilon, \mathrm{rem}}\right)^{2}+\frac{1}{4 \mu \varepsilon^{2}} \int_{-\infty}^{0}\left(\tilde{\pi}_{-}^{C, \varepsilon, \mathrm{rem}}\right)^{2}\right) \\
& \quad+\int_{0}^{1}\left(\partial_{\sigma} \pi_{+, i}^{C, \varepsilon, \mathrm{rem}}\right)^{2} .
\end{aligned}
$$

When we combine the previous inequalities, we obtain for $\varepsilon$ small enough,

$$
\begin{aligned}
\int_{-\infty}^{0}\left(\partial_{\sigma} \tilde{\pi}_{-}^{C, \varepsilon, \mathrm{rem}}\right)^{2}+ & \int_{0}^{1}\left(\partial_{\sigma} \pi_{+, i}^{C, \varepsilon, \mathrm{rem}}\right)^{2}+\int_{1}^{+\infty}\left(\partial_{\sigma} \tilde{\pi}_{+, e}^{C, \varepsilon, \mathrm{rem}}\right)^{2} \\
& +\frac{1}{\mu \varepsilon^{2}} \int_{-\infty}^{0}\left(\tilde{\pi}_{-}^{C, \varepsilon, \mathrm{rem}}\right)^{2}+\frac{1}{2} \int_{1}^{+\infty}\left(\tilde{\pi}_{+, e}^{C, \varepsilon, \mathrm{rem}}\right)^{2} \leq \mathcal{O}\left(\varepsilon^{5}\right)
\end{aligned}
$$

which in turn gives the following controls:

$$
\begin{aligned}
\left\|\tilde{\pi}_{-}^{C, \varepsilon, \text { rem }}\right\|_{L^{2}(]-\infty, 0[)} & =\mathcal{O}\left(\varepsilon^{7 / 2}\right), \\
\left\|\pi_{+, i}^{C, \varepsilon, \operatorname{rem}}\right\|_{L^{2}(] 0,1[)} & =\mathcal{O}\left(\varepsilon^{5 / 2}\right), \\
\left\|\tilde{\pi}_{+, e}^{C, \varepsilon, \operatorname{rem}}\right\|_{L^{2}(1, \infty)} & =\mathcal{O}\left(\varepsilon^{5 / 2}\right),
\end{aligned}
$$

and thus ends the proof of prop ??. 


\subsection{Proof of Lemma??}

Using the expressions of (??) in (??), we can now expand $F(C, \varepsilon)$ :

$$
\begin{aligned}
F(C, \varepsilon)= & \int_{-\infty}^{0} \pi_{-}^{C, \varepsilon}+\int_{0}^{1} \pi_{+, i}^{C, \varepsilon}+\int_{1}^{+\infty} \pi_{+, i}^{C, \varepsilon}+\varepsilon-\mu \varepsilon^{2} \\
= & \int_{-\infty}^{0} \pi_{-}^{C, \varepsilon, \mathrm{rem}}+\int_{0}^{1} \pi_{+, i}^{C, \varepsilon, \mathrm{rem}}+\int_{1}^{+\infty} \pi_{+, i}^{C, \varepsilon, \mathrm{rem}} \\
& +\int_{-\infty}^{0} \pi_{-}^{C, \varepsilon, \mathrm{app}}+\int_{0}^{1} \pi_{+, i}^{C, \varepsilon, \mathrm{app}}+\int_{1}^{+\infty} \pi_{+, i}^{C, \varepsilon, \mathrm{app}}+\varepsilon-\mu \varepsilon^{2} \\
= & \int_{-\infty}^{0} \pi_{-}^{C, \varepsilon, \mathrm{rem}}+\int_{0}^{1} \pi_{+, i}^{C, \varepsilon, \mathrm{rem}}+\int_{1}^{+\infty} \pi_{+, i}^{C, \varepsilon, \mathrm{rem}} \\
& +2 C \mu \varepsilon^{3}+C \varepsilon^{2}+\varepsilon^{3} \int_{0}^{1} \theta(\sigma)\left(1-\exp \left(\frac{\sigma-1}{\mu \varepsilon}\right)\right) \mathrm{d} \sigma+C \varepsilon+\varepsilon-\mu \varepsilon^{2}
\end{aligned}
$$

Let us first note that

$$
\sup _{\sigma \in[0,1]}\left|\theta(\sigma)\left(1-\exp \left(\frac{\sigma-1}{\mu \varepsilon}\right)\right)\right| \leq 1,
$$

so that

$$
\varepsilon^{3} \int_{0}^{1} \theta(\sigma)\left(1-\exp \left(\frac{\sigma-1}{\mu \varepsilon}\right)\right) \mathrm{d} \sigma=\mathcal{O}\left(\varepsilon^{3}\right) .
$$

We now estimate the integrals of $\pi_{-}^{C, \varepsilon, \mathrm{rem}}, \pi_{+, i}^{C, \varepsilon, \mathrm{rem}}$ and $\pi_{+, e}^{C, \varepsilon, \mathrm{rem}}$. To achieve this, we use prop ?? which gives us the order of the respective $\mathrm{L}^{2}$ norms of $\widetilde{\pi}_{-}^{C, \varepsilon, \mathrm{rem}}$ and $\widetilde{\pi}_{+, e}^{C, \varepsilon \text { rem }}$. Indeed we use Hölder inequality to get the following controls,

$$
\begin{aligned}
\int_{-\infty}^{0} \pi_{-}^{C, \varepsilon, \mathrm{rem}} & =\int_{-\infty}^{0} \exp \left(\frac{\sigma}{\mu \varepsilon}\right) \widetilde{\pi}_{-}^{C, \varepsilon, \mathrm{rem}} \\
& \lesssim \varepsilon^{1 / 2}\left\|\widetilde{\pi}_{-}^{C, \varepsilon, \mathrm{rem}}\right\|_{\mathrm{L}^{2}(]-\infty, 0[)} \lesssim \mathcal{O}\left(\varepsilon^{3}\right), \\
\int_{0}^{1} \pi_{+, i}^{C, \varepsilon, \mathrm{rem}} & \lesssim\left\|\pi^{C, \varepsilon, \mathrm{rem}}\right\|_{\left.\left.\mathrm{L}^{2}(] 0,1\right]\right)} \lesssim \mathcal{O}\left(\varepsilon^{5 / 2}\right), \\
\int_{1}^{+\infty} \pi_{+, e}^{C, \varepsilon, \mathrm{rem}} & =\int_{1}^{+\infty} \exp \left(\frac{\varepsilon}{2}(\sigma-1)\right) \widetilde{\pi}_{+, e}^{C, \varepsilon, \mathrm{rem}} \\
& \lesssim \frac{1}{\varepsilon^{1 / 2}}\left\|\widetilde{\pi}_{+, e}^{C, \varepsilon, \mathrm{rem}}\right\|_{\mathrm{L}^{2}(1, \infty)} \lesssim \mathcal{O}\left(\varepsilon^{2}\right) .
\end{aligned}
$$

since, by explicit integration, we get

$$
\begin{gathered}
\left\|\exp \left(\frac{\cdot}{2 \mu \varepsilon}\right)\right\|_{L^{2}(]-\infty, 0[)}=(\mu \varepsilon)^{1 / 2}, \\
\left\|\exp \left(\frac{\varepsilon}{2}(\cdot-1)\right)\right\|_{L^{2}(+1, \infty)}=\frac{1}{\varepsilon^{1 / 2}},
\end{gathered}
$$

Finally we have

$$
\int_{-\infty}^{0} \pi_{-}^{C, \varepsilon, \mathrm{rem}}+\int_{0}^{1} \pi_{+, i}^{C, \varepsilon, \mathrm{rem}}+\int_{1}^{+\infty} \pi_{+, i}^{C, \varepsilon, \mathrm{rem}}=\mathcal{O}\left(\varepsilon^{2}\right) .
$$


Now we use (??) and (??) in (??) to obtain:

$$
F(C, \varepsilon)=(C+1) \varepsilon+\mathcal{O}\left(\varepsilon^{2}\right),
$$

which is exactly what prop ?? states

Ackknowledgements. The author would like to thank DiDIER BRESCH and MARGUERITE GISCLON for making the paper readable in the first place. This work was partially supported by the FP7-REGPOT-2009-1 project "Archimedes Center for Modeling, Analysis and Computation" and by the ANR-08-SYSC-010 project "Maniphyc".

\section{References}

[1] Mohamed Ben Alaya and Benjamin Jourdain. Probabilistic approximation of a nonlinear parabolic equation occurring in rheology. J. Appl. Probab., 44(2):528-546, 2007.

[2] Éric Cancès, Isabelle Catto, and Yousra Gati. Mathematical analysis of a nonlinear parabolic equation arising in the modelling of non-Newtonian flows. SIAM J. Math. Anal., 37(1):60-82 (electronic), 2005.

[3] Jean-Yves Chemin, Benoît Desjardins, Isabelle Gallagher, and Emmanuel Grenier. Mathematical geophysics, volume 32 of Oxford Lecture Series in Mathematics and its Applications. The Clarendon Press Oxford University Press, Oxford, 2006. An introduction to rotating fluids and the Navier-Stokes equations.

[4] Pascal Hébraud and François Lequeux. Mode-coupling theory for the pasty rheology of soft glassy materials. Phys. Rev. Lett., 81(14):2934-2937, Oct 1998.

[5] Tosio Kato. Perturbation theory for linear operators. Classics in Mathematics. Springer-Verlag, Berlin, 1995. Reprint of the 1980 edition.

[6] Julien Olivier. Asymptotic analysis in flow curves for a model of soft glassy rheology. Z. Angew. Math. Phys, 61(3):445-466, 2010.

[7] Julien Olivier and Michael Renardy. On the generalization of the hébraudlequeux to multidimensional flows. In preparation.

[8] Julien Olivier and Michael Renardy. Glass transition seen through asymptotic expansions. SIAM J. Appl. Math., 71(4):1144-1167, 2011.

[9] Patrick Oswald. Rhéophysique. Échelles. Belin, Paris, 2005. 